\section{HDACs get complex}

\section{By Joanne Kotz, Senior Editor}

Cellzome AG has reported a chemical proteomic method for determining the selectivity of HDAC inhibitors. The approach makes it possible, for the first time, to determine the effects of an inhibitor on HDACs as components of naturally occurring protein complexes rather than as the less biologically relevant isolated enzymes. ${ }^{1}$ The company is already exploring the potential of the method to identify HDAC inhibitors that are more selective and less toxic than the array of molecules currently on the market or in the clinic.

Histone deacetylases (HDACs) are a family of enzymes that regulate gene expression by removing acetyl groups from histones. In addition, HDACs regulate a variety of other cellular processes through the deacetylation of nonhistone proteins.

There are two marketed HDAC inhibitors: Merck \& Co. Inc.'s Zolinza vorinostat and Celgene Corp.'s Istodax romidepsin. Both drugs are marketed for cutaneous $\mathrm{T}$ cell lymphoma (CTCL). At least 16 other companies have HDAC inhibitors in clinical trials, mostly for cancer.

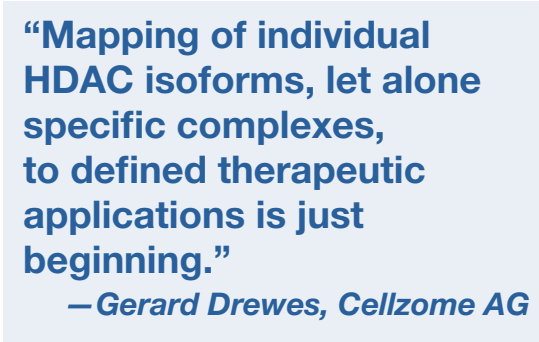

centration increased to a level at which it competed with the chemical probe, it inhibited binding of the HDAC complexes to the beads. The $\mathrm{IC}_{50}$ value of the inhibitor for each particular HDAC complex could be determined using mass spectrometry to quantify changes in bead-bound HDACs.

As proof of concept, the Cellzome team used the technology to characterize some HDAC inhibitors in clinical development. In cell lysates from a human myelogenous leukemia cell line, 267 proteins-including 6 HDACs and 29 proteins known to form complexes with HDACs-bound to the chemical probes. The researchers titrated 16 chemically diverse HDAC inhibitors, including both approved drugs, with the bead-bound HDAC complexes and determined their $\mathrm{IC}_{50}$ values.

The study revealed the surprising result that a single inhibitor could have a different effect on distinct protein complexes containing the same HDAC isoforms.

Data were published in Nature Biotechnology.

"It's generally believed that the toxicities of HDAC inhibitors in the clinic are the result of nonselectivity. However, up until recently, whether [we thought] an inhibitor is isoform-selective or nonselective depended a lot on the assays and dosage of the inhibitor," said Ed Seto, chair of the Department of Molecular Oncology at the H. Lee Moffitt Cancer Center \& Research Institute. "Thus, until we know which inhibitors are truly isoform- or complex-selective, and test them in hypothesis-driven clinical trials, the jury is still out. The work presented in this paper using HDAC complexes as targets to dis-

However, the majority of these compounds are nonselective, inhibiting more than 1 of the 11 HDACs. ${ }^{2}$ For example, the two approved drugs both inhibit at least four HDAC isoforms.

As a result, the precise HDAC target for an inhibitor's observed therapeutic effect-and its side effects-has been difficult to determine, said Gerard Drewes, VP of discovery research at Cellzome.

"Which targets will be best for which indication is still largely unknown," he told SciBX.

Further complicating the matter is that HDACs are found in large protein complexes within cells, and a single HDAC can play different biological roles in different complexes.

"Mapping of individual HDAC isoforms, let alone specific complexes, to defined therapeutic applications is just beginning," said Drewes.

To develop more selective HDAC inhibitors, Cellzome decided to adapt a mass spectrometry-based chemical proteomics method the company had originally developed for determining the selectivity of kinase inhibitors. ${ }^{3}$

First, the researchers generated chemical probes consisting of a nonselective HDAC inhibitor attached to sepharose beads. When added to cell lysates, the probes bound any protein complexes containing HDACs, thus capturing them on the beads.

Before the team captured the HDAC complexes on beads, they titrated cell extracts with different inhibitors. As the inhibitor con- cover selectivity is an excellent start."

"Studies such as this further underscore the pressing need for enzyme-specific-and now perhaps complex-specific-HDAC inhibitors as chemical probes and lead therapeutic agents," added James Bradner, an investigator in the Department of Medical Oncology and staff physician in the Stem Cell Transplantation and Hematologic Malignancies Services at the Dana-Farber Cancer Institute.

"Highly selective compounds would be expected to extend the therapeutic window of this promising class of agents," said Bradner, who also is a cofounder of Acetylon Pharmaceuticals Inc., a developer of HDACtargeted therapeutics.

He added that the approach could be used to explore subtle differences observed across tissues to shed light on the efficacy and toxicity of investigational agents.

Acetylon has ACY-1215, an HDAC6 inhibitor, in preclinical development for multiple myeloma.

"One of the reasons for the apparent low single-agent activity of the current HDAC inhibitors in solid tumor clinical trials may be the dose and schedule limitations due to the side effects," said Sriram Balasubramanian, senior director of translational medicine and HDAC project leader at Pharmacyclics Inc. "Isoform-selective inhibitors could provide better exposure and higher dose levels, which might lead to better efficacy." 


\section{ANALYSIS}

Pharmacyclics' PCI-24781, a broad-spectrum HDAC inhibitor, is in Phase I/II testing for solid tumors and hematological cancers. The company also has HDAC8-selective inhibitors in preclinical testing.

"This paper provides a good method to screen small molecules against HDACs in their active forms and perhaps even develop complex-specific inhibitors," said Balasubramanian.

\section{Getting specific}

Next up for Cellzome will be developing selective inhibitors for HDACs and other epigenetic targets. Cellzome did not disclose which HDACs would be initially targeted. Drewes told SciBX that the method could potentially broaden the chemical space available for developing HDAC inhibitors. "It is speculative but possible that with this assay you could uncover new chemical scaffolds, and the assay can definitely be used to optimize current scaffolds," he said.

Other researchers also see opportunities.

Kevin Lee, VP and head of GlaxoSmithKline plc's EpiNova DPU in the Immuno-Inflammation Centre of Excellence for Drug Discovery, noted that HDAC9 looks interesting for the potential treatment of immune disease.

Balasubramanian said HDAC2-selective inhibitors may have particular potential in solid tumors, whereas HDAC6- and HDAC8-selective inhibitors look promising in hematological indications.

"Highly selective inhibitors of HDAC3 would be of substantial interest to the cancer and metabolism research fields," added Bradner.

Drewes said Cellzome is open to partnering in the HDAC space, particularly in cancer and inflammation.

Under a March 2010 deal, Cellzome and GSK are using a similar chemical proteomic platform to the one described in Nature Biotechnology to identify small molecule drug leads against four undisclosed epigenetic target classes for immuno-inflammation indications.

According to Drewes, the four targets do not include HDACs.

Kotz, J. SciBX 4(4); doi:10.1038/scibx.2011.91

Published online Jan. 27, 2011

\section{REFERENCES}

1. Bantscheff, M. et al. Nat. Biotechnol.; published online Jan. 23, 2011; doi:10.1038/nbt.1759

Contact: Gerard Drewes, Cellzome AG, Heidelberg, Germany e-mail: gerard.drewes@cellzome.com

Contact: Marcus Bantscheff, same affiliation as above e-mail: marcus.bantscheff@cellzome.com

2. Balasubramanian, S. et al. Cancer Lett. 280, 211-221 (2009)

3. Bantscheff, M. et al. Nat. Biotechnol. 25, 1035-1044 (2007)

COMPANIES AND INSTITUTIONS MENTIONED

Acetylon Pharmaceuticals Inc., Winchester, Mass.

Celgene Corp. (NASDAQ:CELG), Summit, N.J.

Cellzome AG, Heidelberg, Germany.

Dana-Farber Cancer Institute, Boston, Mass.

GlaxoSmithKline plc (LSE:GSK; NYSE:GSK), London, U.K.

H. Lee Moffitt Cancer Center \& Research Institute, Tampa, Fla.

Merck \& Co. Inc. (NYSE:MRK), Whitehouse Station, N.J.

Pharmacyclics Inc. (NASDAQ:PCYC), Sunnyvale, Calif. 